\title{
An Energy Neutral Wearable Camera with EPD Display
}

\author{
Jetmir Nehani \\ University of Bologna \\ $\mathrm{DEl}$, Unibo \\ Bologna, Italy \\ jetmir.nehani@unibo.it
}

\author{
Davide Brunelli \\ University of Trento \\ DII \\ Trento, Italy \\ davide.brunelli@unitn.it
}

\author{
Michele Magno, \\ Lukas Sigrist \\ and Luca Benini \\ IIS, ETH \\ Zurich, Switzerland \\ magnom@iis.ee.ethz.ch \\ lukas.sigrist@tik.ee.ethz.ch \\ lbenini@iis.ee.ethz.ch
}

\begin{abstract}
Wearable technologies are flooding the consumer market, and have massively entered the market of electronic consumers and people are now surrounded by an increasing number of "smart" objects to wear. The main issue that limits the success of these devices is limited battery lifetime. Energy-neutral operation, which does not require battery recharging or replacement (similar to automatic quartz watches) is highly desirable in this context. In this paper, we present the first energy-neutral wearable device, equipped with an ultra low power camera and an electrophoretic display (EPD) which is supplied by a solar energy harvester. The novel design includes several hardware and software optimizations to achieve energy neutrality. In particular, we implemented innovative methods for displaying gray-scale images to obtain up to 9 gray-scale levels using a black-andwhite display. This reduces by $43.7 \%$ the energy consumption in comparison to the state of art. Moreover, we implemented aggressive power management for the camera acquisition which saves up to $91.4 \%$ of energy to acquire an image. Experimental results, with different scenarios, demonstrate advanced functionality and the energy neutrality of the system that can acquire and display up to 54 images per hour in indoor scenario.
\end{abstract}

\section{Categories and Subject Descriptors}

J.7 [Computer Applications]: COMPUTERS IN OTHER SYSTEMS-Consumer products

\section{Keywords}

wearable devices; energy neutral; electrophoretic displays; power optimization; energy harvesting; low power camera.

\section{INTRODUCTION}

Wearable devices have quickly gained popularity over the last few years. Today almost all major consumer electronics manufacturers are working on wearable products or already have similar devices on the market. People are wearing everything that can be connected to the internet, e.g.

Permission to make digital or hard copies of all or part of this work for personal or classroom use is granted without fee provided that copies are not made or distributed for profit or commercial advantage and that copies bear this notice and the full citation on the first page. Copyrights for components of this work owned by others than ACM must be honored. Abstracting with credit is permitted. To copy otherwise, or republish, to post on servers or to redistribute to lists, requires prior specific permission and/or a fee. Request permissions from Permissions@ acm.org. WearSys'15, May 18, 2015, Florence, Italy.

Copyright (C) 2015 ACM 978-1-4503-3500-3/15/05 ...\$15.00

http://dx.doi.org/10.1145/2753509.2753510 from clothes that light up when users receive phone calls (Bluetooth-enabled dresses) to smart glasses (e.g. Google glass) and smart watches ${ }^{1}$ (apple watch from Apple). Most of the biggest hi-tech companies will enter into the market as soon as they can guarantee at least 24 hours of energy autonomy before battery recharging.

Due to time-to-market constraints, costs, and energy issues, present-day wearable devices are mainly battery-powered and can only achieve multiple-day autonomy by limiting their computational capabilities; when executing complex functionality, they need to be recharged frequently. This confirms that all the present wearable products miss the expectations for a truly unobtrusive user experience, and that the most critical challenge in designing such devices, is the autonomy of the system ${ }^{2}$. Chip makers are focused on developing new components, microcontrollers and sensors that have low power consumption and are devoting a considerable research effort to find hardware-software solutions to optimize or extend the lifetime of these devices. Power management for wearable devices has been proposed in several forms and shares challenges typical of networked embedded system (i.e. WSN)[1, 2]. Hardware-software approaches are focused on power management such as dynamic voltage and frequency scaling, power-aware scheduling, dynamic power management by using low-power modes, etc. Alternative approaches are based on hardware choices such as use of low-power subsystems (sensors, microcontrollers, memories, etc,) or custom ultra-low power hardware components $[3,4$, $5,6]$.

For years, displays have been considered a power hungry subsystem, in particular if equipped with back-lights technology. Today some classes of displays permit huge energy saving, thanks to electrophoretic technology, and they are becoming appealing for the wearable/mobile market. Nevertheless, there is still a large margin to reduce further the consumption of the displays and to render new images with an energy budget of only a few pJ, because they require no power to retain an image once rendered. The EPD technology is based on the phenomenon of electrophoresis, which rearranges charged pigment particles using an applied electric field. EPDs [7] are used on e-readers, smart watches, mobile phones, tag prices, etc. They need power only during the updating phase of the display and thanks to their bi-stable nature; zero energy is required to maintain images on the screen for very long periods (i.e. weeks, months). Furthermore, EPDs, also called E-papers, use a reflective display technology that does not require internal light source and

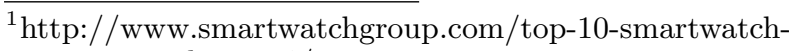
companies-sales-2014/

${ }^{2}$ http://embedded-computing.com/articles/cutting-thecord-energy-harvesting-in-wearables/
} 
permits a large range of view. Monochrome, grayscale and color EPDs are already available on the market with different costs and features. Color and Grayscale ones have a refresh rate time that is greater than monochrome EPDs. The grayscale levels available in EPDs are usually 16, while color displays feature 4096 levels of color and need dedicated controllers $[8,9]$. Higher refresh rate and dedicated controller increase both the performance and power consumption of color displays which are still not suitable for ultra low power devices.

Another interesting novel low power technology in memory domain is Ferroelectric RAM (FRAM)[10]. This memory permits to storage non-volatile information with a speed very close to SRAM memory. Non-volatile memories are very useful in systems where the power supply can be intermittent, such as the devices supplied by energy harvesters [10]. Thus, power management can check if the MCU can be completely turn-off when the energy level is low and save power and then to turn-on, restoring the last saved context of the registers.

In this paper, we combine the benefits of EPDs and FRAM technologies, coupled with hardware and software optimization to minimize the power consumption of the whole device. Thus, we integrate an ultra low power microcontroller with on board FRAM, an EPD and a low power camera sensor in a whole device equipped with solar energy harvester with a high-efficiency energy conversion circuit to achieve indefinite sustainability. We propose an alternative and more efficient mode of operation with respect to the standard suggested by manufacturers for the EPD, which reduces the update time to display an image. Due to this optimization, the overall energy of the device is decreased. Furthermore, we extended the mode to generate grayscale levels, and we compared three different methods with different trade-offs between quality of the images and energy used to be adopted to achieve energy neutrality. Experimental results demonstrate the energy neutrality of the device based on different implemented scenarios.

The remainder of this paper is organized as the follows: Section 2 describes the recent related work in this area, in Section 3 the architecture of the device and the details of the sensors are presented. In Section 4 the power optimization on EPD is depicted and the methods for displays grayscale images are described. In section 6 the analog camera sensor and the power optimization are presented. In Section 7 the harvester is described, while experimental results are presented in Section 8. Finally, Section 9 concludes this paper.

\section{RELATED WORK}

Portable wearable devices have been studied and reported several times in academic communities. Most of them are equipped with batteries and are developed for a specific application $[11,12]$. There are different proposed examples of wearable devices with sensors for health care applications $[13,14,15]$. A typical sensing device consists of a batterypowered computing unit, a wireless communication interface, one or more sensors and power supply packaged in a small and unobtrusive form factor suitable to be attached to the human body [16, 17]. Thus, low power design is even more challenging in wearable systems due to the limited energy availability of the battery. In fact, although integrated circuits have significantly improved their energy efficiency thanks to Moore's law, battery technology has not made similar strides. Thus, low-power design alone is not enough to make these devices wearable with battery lifetimes of months or years and many promising works tried to extend the life time with wearable energy harvesting[18]. Traditionally, radio communication has been reported as the primary consumer of energy in a wearable product and many

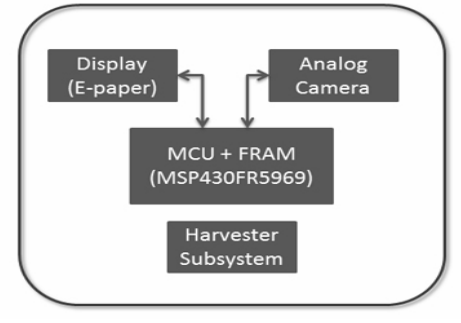

Figure 1: Hardware architecture of the wearable device.

power management policies have been investigated to reduce the impact of the wireless communication [19]. However, many application scenarios require ad-hoc sensors ${ }^{3}$, often leading to sensor nodes consuming more power during data acquisition and computation/processing phases rather than during communication [14] and camera sensors are an example. Moreover, "local intelligence" algorithms in general require more power than what is typically available from energy harvesting. Thus, the power requirements of these sensors are generally tens to hundreds of $\mathrm{mW}$ range, which is similar to (or often higher) the transceiver's power consumption[14]. On-board processing and hardware software optimizations can save energy due to the reduction of the data transmission. Thus, efficient power management techniques that minimize the activity/processing of energy-consuming sensors are necessary. For this reason, the use of low-dutycycle data acquisition and sleep-cycle algorithms can considerably increase the lifetime of wearable devices [20]. A node can generally be supplied for only a few hours at 100 duty cycle with a standard battery, and therefore these optimization techniques are essential.

Displays are another power hungry subsystem, and EPDs devices are trying to make them suitable for low power wearable devices. To date, experimental characterizations of EPDs and power reduction have been presented [21], but they mainly focus on technology improvements and not on driver optimization. In this paper, we propose an alternative and more efficient mode to use the EPD in comparison with the standard suggested by manufacturers, which reduces the power consumption. Furthermore, we extended the mode to generate grayscale levels, where we achieve more grayscale levels than the ones described in [21].

\section{ARCHITECTURE}

The device, presented in this paper, consists of a wearable device with an ultra low power camera, an e-paper display and a solar energy harvester where the computational capabilities are provided by a microcontroller. The block diagram architecture of the presented system includes an energy harvester subsystem, which converts and accumulates energy as shown in Fig. 1. The microcontroller acquires the data from the analog camera through its internal Analog Digital Converter and then stores them in the FRAM memory. The acquired images can be displayed on the e-paper display on demand.

\subsection{Microcontroller}

The specific microcontroller used in our design is the MSP430FR5969 from Texas Instruments TI which has integrated $64 \mathrm{~KB}$ of non-volatile FRAM. The maximum CPU frequency is $16 \mathrm{MHz}$ with the possibility to dynamically decrease the

\footnotetext{
${ }^{3}$ http://inhabitat.com/hybrid-power-bracelet-runs-onsolar-and-thermal-energy/fujistu/
} 
frequency down to $32 \mathrm{KHz}$ to reduce the power consumption. The microcontroller provides one active mode and 7 different power saving modes called Low Power Modes (LPM) which allow the processor to reduce power consumption, while its peripherals are still working. In active mode, the current consumption of the microcontroller is around $100 \mu \mathrm{A} / \mathrm{MHz}$ and in deep sleep mode (LPM4.5) the current consumption is only $20 \mathrm{nA}$. Finally, the microcontroller integrates several peripherals such as a 12 bit ADC module, timers with PWM, Direct Memory Access (DMA) module and three eUSCI modules to implement SPI, UART and I2C serial communication. The ADC is used to acquire the image from the camera while the display is updated by the SPI interface communication.

\section{E-PAPER DISPLAY AND POWER OPTI- MIZATION}

An EPD panel developed from Pervasive Display inc. PDI has been used in this work. The EDP consists of a black and white E-Ink film on the top and an active-matrix TFT (Thin Film Transistor) to drive the microcapsules [22]. The EPD has 128 x 96 pixels, and each of them is driven by an active matrix of row and column electrodes. The EPD driver writes one row at s time, by applying a modulated electric potential on each electrodes, which moves the pigmented particles and generate the image.

The MSP430 microcontroller firmware generates the signals for a correct image refresh process which consists of 4 steps and the plot in Figure 2 a) shows an entire EPD update cycle. During the first step, the display is powered on. This step is short and the peak consumption is necessary for charging the internal switching capacitors used by the display. In the second step, the microcontroller generates all the necessary signals for initializing the driver, such as setting temperature factor to the controller, determining the correct EPD type and correct voltage threshold. The real process of moving pigment particles and displaying a new image is realized in Step 3. Typically this step contains four different phases (or stages): i) the inverse of the previous image is printed on the display; ii) then a complete white background is printed; iii) then the inverse of the new image is temporary overprinted, iv) and eventually the new image overwrites everything. These four stages are not necessary, but they are usually implemented as a good rule to guarantee that all the particles are moved during each image refresh and to eliminate the ghosting effect $[9,22]$. Each stage has a reserved time slot that is usually calculated taking into account the operating temperature.

In this work we considered a time interval of $480 \mathrm{~ms}$, which is adequate for a temperature range $0-30{ }^{\circ} \mathrm{C}$. Within $480 \mathrm{~ms}$ assigned to each phase, about 13 frames can be over-written repeatedly. This permits to move the particles on the top of the plane according to the black or white value of the image pixel. Finally, the fourth Step disconnects the electrodes and switches the power supply of the display off. The complete process for updating the EPD takes $2660 \mathrm{~ms}$. Step 3 takes a large part of the time $(1920 \mathrm{~ms})$ and contributes remarkably to the total energy consumption which is $24.5 \mathrm{~mJ}$. An optimized version of [23] has been implemented in this work to save energy. This optimization consists on comparing different combination of phases inside Step 3; and implementing only the phase that overwrites new images. In addition, we reduce switching time from $480 \mathrm{~ms}$ to $360 \mathrm{~ms}$, which was tested to be still an optimal value in the operating temperature range. The new $360 \mathrm{~ms}$ interval causes the reduction of number of frames, which can be sequentially written on the EPD, down to 10 (i.e. $36 \mathrm{~ms}$ each).

To test the image quality of the proposed method with re- a)

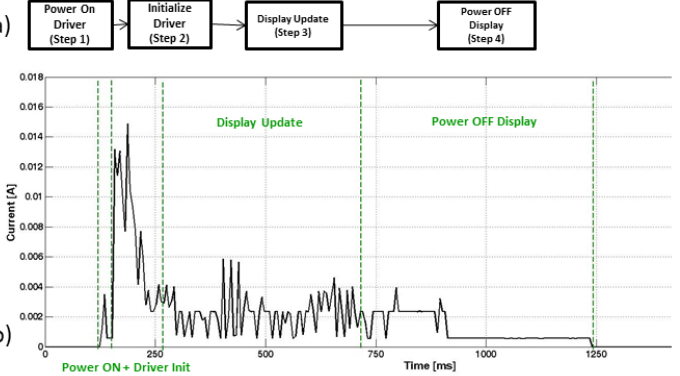

Figure 2: a) Steps that are necessary during an EPD update cycle. b) Current consumption during an EPD update cycle.

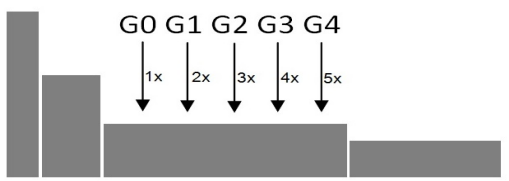

Figure 3: Grayscale images obtained with Method 1.

spect the traditional one, we use an image comparison utility that makes use of a computational model of the human visual system to compare two images [24]. In this case, we have assessed that the image visualized on display has the same perceptual quality of the traditional use of EPD $(<$ $1.5 \%$ difference). Figure $2 \mathrm{~b}$ ) plots the measured consumption of the complete EPD update cycle. The total time for updating the EPD is reduced about $60 \%$ and the total energy consumption is $10.7 \mathrm{~mJ}, 56.3 \%$ less than the baseline.

\section{GRAYSCALE ENHANCEMENT ON BLACK-AND-WHITE EPD}

Although grayscale and color EPDs are available on the market, the low cost and especially the power consumption of BW displays make these devices particularly appealing. Other 3 methods has been implemented to extend the previous low power approach for generating grayscale images on BW displays. An analytic comparison of the quality of the images and of the energy consumed is provided in Table 2. All these methods manage the number and the content of the frames written during Step 3 and starting from a white background.

Method 1: Grayscale pixels are obtained varying the number of write operations of black pigment particles. The greater is the number of frames of the corresponding black particles; the darker is the pixel that we want to obtain. Since the time necessary to write a frame is $36 \mathrm{~ms}$, grayscale levels are realized by dividing the image in overlapping layers. Each layer correspond to a grayscale level. We drive only the black particles writing in total 5 layers and we obtain 5 grayscale (G0 to G4) levels during step 3 as illustrated in Figure 3. Each frame Fx will move on foreground only the black particles of the pixel which must have a gray intensity greater than the corresponding Grayscale level Gx. (Gpixel $>\mathrm{Gx}$ ). This method is very fast with minimum energy expenditure, but can generate only 5 grayscale levels Figure $6 a)$. Increasing the number of frame does not produce any additional appreciable gray level.

Method 2: Grayscale levels are realized by running multiple times all the four steps as depicted in Figure 4. With this 


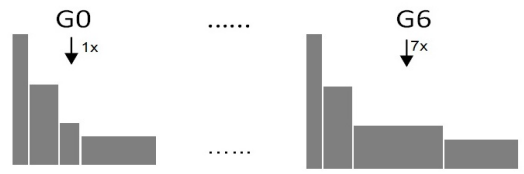

Figure 4: Grayscale images obtained with Method 2.

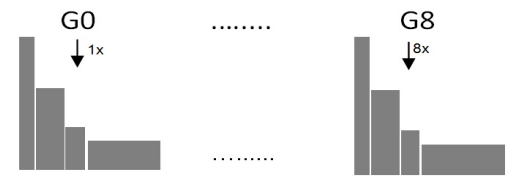

Figure 5: Grayscale images obtained with Method 3.

method, the interval dedicated to Step 3 is incremented each cycle, depending on the grey pixel to obtain. Like in Method 1 , we manage only the black pixels and we can achieve up to 7 visible and recognizable grayscale levels (G0 to G6) as shown in Figure 6b). Method 3: The grayscale levels are realized running multiple times all the four steps. Unlike the previously described methods, here also white pigment particles are managed and contribute to create a gray level. This allows to minimize the time dedicated by Step 3, which is kept constant at $36 \mathrm{~ms}$, as shown in Figure 5. The EPD is updated frame by frame with fixed time frame and we managed to obtain up to 9 distinct grayscale levels (G0 to G8), reported in Figure 6c), which are $2 \mathrm{x}$ better than what is achieved in the literature [25].

\section{ANALOG CAMERA}

An analog grayscale camera from Centeye with Stonyman chip has been used as a low power and low resolution image sensor. The Stonyman chip contains a $112 \mathrm{x}$ 112 pixels arranged in a conventional square array and consumes only $2 \mathrm{~mW}$ at $3 \mathrm{~V}$. The logarithmic voltage response of the shutterless pixels are directly connected to the analog output. The camera requires an interface with five digital inputs and one analog output that are connected to the microcontroller. The digital inputs are pulsed according to an intuitive and easy to understand sequence, and the ana$\log$ output contains the analog pixel signal which need to be digitized by the integrated $\mathrm{ADC}$ of the microcontroller. The digital data is stored in the integrated FRAM of the microcontroller. Moreover, the digital inputs allow the microcontroller to read out the entire image or select a subarea of the image. Two methods have been developed to acquire an entire image from the camera.

Method 1 : The digital inputs are generated by the CPU of the microcontroller. These signals configure the camera to read out a complete image. The analog output of the camera contains the analog pixel that are digitized from the ADC. Then the CPU stores each pixel in the FRAM. The time for acquiring one image is $231 \mathrm{~ms}$, and the consumed energy by camera and microcontroller is $2.63 \mathrm{~mJ}$. The energy consumed by the camera during the acquisition is $417 \mu \mathrm{J}$.

Method 2 : The second method implemented acquires an image with even less energy. In this optimization, the digital

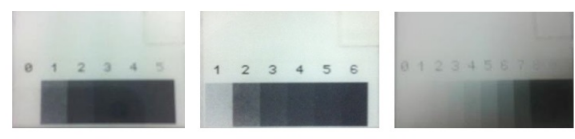

Figure 6: Results of grayscale level with the three methods
Table 1: Time and energy consumption for one image acquisition

\begin{tabular}{|c|c|c|}
\hline Method & Time $(\mathrm{ms})$ & Energy $(\mathrm{mJ})$ \\
\hline Method 1 & 231 & 2.63 \\
\hline Method 2 & 47 & 0.226 \\
\hline
\end{tabular}

inputs are generated by the integrated timers of the microcontroller while the data is transferred to the FRAM using the DMA module. The acquisition time is only $47 \mathrm{~ms}$ and the total energy consumed is $226 \mu \mathrm{J}$. The total energy consumed by the camera chip is $64 \mu \mathrm{J}$.

As shown on Table 1, there is a significant improvement in terms of time and consumed energy, when acquiring an image with method 2 instead of method 1 . We have reduced the time to acquire an image by a factor of 5 and the energy for one image acquisition by $91.4 \%$.

\section{ENERGY HARVESTER}

In order for the system to achieve energy neutrality, we integrated eight small amorphous silicon solar cells, AM1471CA from Sanyo Energy ${ }^{4}$, connected in parallel. These solar cells can delivery up to $100 \mu \mathrm{A} @ 1.9 \mathrm{~V}$ under office light conditions and up to $15 \mathrm{~mA} @ 1.6 \mathrm{~V}$ under sunlight which can satisfy the power budget of the above mentioned device. The conversion system is based on the BQ25570 ultra lowpower IC from Texas Instruments, which includes an algorithm to perform a maximum power point tracking (MPPT) and configurable protection of the storage from the undervoltage and over-voltage events. The BQ25570 exploits a high efficient boost converter to harvester energy from the eight solar cells and it can recharge the supercapacitors or battery. During the activity the BQ25570 consumes less than $500 \mathrm{nA}$ and about $5 \mathrm{nA}$ when it is turned-off.

\section{EXPERIMENTAL RESULTS}

In this section we present experimental evaluations in terms of power consumption, energy harvester and power management. We measured the power consumption of the device and its subsystems in different states. In addition we performed an experimental evaluation and tests to determine the efficiency of the energy harvesting subsystem. First we measured the energy consumed by the EPD that is calculated according to the following formula.

$$
E=\sum_{i=0}^{N} P_{i} \cdot \Delta t_{i}
$$

where $E$ is the energy consumption, $N$ is the total number of the frames for EPD updating cycle, $P_{i}$ is the average of the power consumption for each cycle, and $\Delta t_{i}$ is the update time of the EPD. The total energy consumption measured during the standard use of the EPD is $24.5 \mathrm{~mJ}$. The power optimization on black and white EPD, proposed in section 4 of this work, achieves an energy saving of $56.3 \%$, with only $10.7 \mathrm{~mJ}$ necessary to refresh an image.

Considering the grayscale enhancements, the trade-off between quality of the image and energy expenditure is reported in Table 2. Method 1 is much faster than the others two grayscale methods and saves up to $5 \mathrm{x}$ the energy of the Method 2. However, the range of possible gray intensities is quite limited. Method 3 appears to have the best trade-off between quality of the image and energy expenditure, with the higher pixel dynamic and a consumption which is lower than Method 2. An interesting approach could be adopting an adaptive management which switches between Method 1 and 3 according to the energy budget and the desired level

\footnotetext{
${ }^{4}$ http://panasonic.net/energy/amorton/en/products/spec/
} AM-1417.html 
Table 2: Grayscale levels, energy consumption and update time for black-and-white and the three grayscale implemented methods

\begin{tabular}{|c|c|c|c|}
\hline Method & $\begin{array}{c}\text { Grayscale } \\
\text { Levels }\end{array}$ & $\begin{array}{c}\text { Update Time } \\
(\mathrm{ms})\end{array}$ & $\begin{array}{c}\text { Energy } \\
(\mathrm{mJ})\end{array}$ \\
\hline Black-White & 2 & 1100 & 10.7 \\
\hline Method 1 & 5 & 1100 & 11.52 \\
\hline Method 2 & 7 & 5050 & 59.00 \\
\hline Method 3 & 9 & 4500 & 48.7 \\
\hline
\end{tabular}

Energy harvested per day and averaged Power per hour

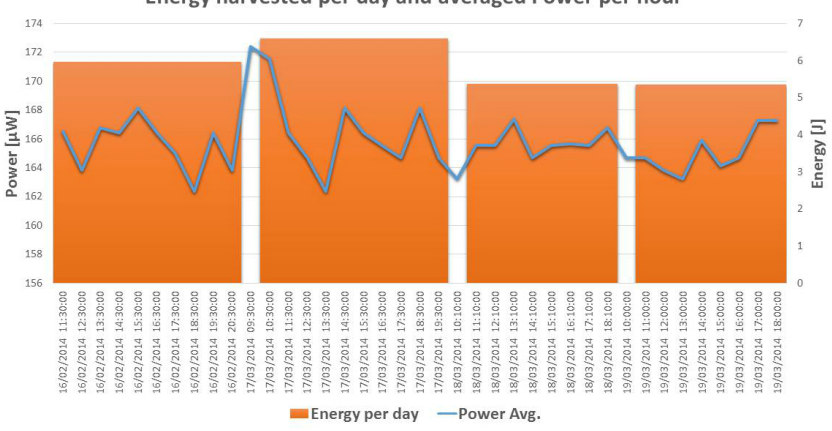

Figure 7: Intake Energy per day accumulated during the four consecutive days acquisition from 11:00am to $7: 00 \mathrm{pm}$ and the averaged power per hour.

of gray in a particular image to display. As presented in Section 6, we have proposed two methods for acquiring images from the analog camera. The second method proposed, achieved an energy saving of $11 \mathrm{x}$, with only $226 \mu \mathrm{J}$ necessary to acquire an image. After measuring the energy consumed by the device, we have evaluated the energy harvested by the solar panels when they are worn. We performed a set of measurements to evaluate the energy stored during four consecutive days in our lab from 11:00am to 07:00pm in indoor scenario. The indoor scenario has been selected to proof the energy neutral capability also in a challenging scenario where the energy available is low and the person is directly wearing the device, meaning it is a realistic scenario (i.e. shadows can cover part of the panels). Figure 7 shows the measured intake energy per day and the averaged power per hour. The total energy accumulated in four consecutive days by the harvester system was $23.27 \mathrm{~J}$ with an average of $5.81 \mathrm{~J}$ per day and $0.6 \mathrm{~J}$ per hour.

To estimate the energy neutrality of the device in different optimization configurations, we estimate the consumed energy by the device, which is calculated by the following formula:

$$
E_{\text {consumed }}=A_{\text {ev } 1} * E_{\text {camera }}+A_{\text {ev } 2} * E_{E P D}
$$

Where $E_{\text {camera }}$ and $E_{E P D}$ are the energies needed to perform the different tasks for Image Acquisition and EPD Update respectively. The $A_{e v 1}$ and $A_{e v 2}$ are the number of times that this task is performed in one hour. The condition of energy neutrality is satisfied when the following equation is true for each period of time:

$$
E_{\text {consumed }}=E_{h}
$$

Where $E_{h}$ is the averaged intake energy accumulated by the harvester, and $E_{\text {consumed }}$ is the energy consumed by the device. In our evaluation we want to determine the $A_{e v 1}$ and $A_{e v 2}$ according to different scenarios when the energy neutrality is achieved. Four scenarios with different EPD implementations have been used to evaluate the benefits of our above mentioned optimizations in terms of the number of acquired and displayed images per hour in an energy neutral environment. We evaluated each of the four scenarios
Table 3: Maximum number of activities per hour with and without camera optimization for the four scenarios

\begin{tabular}{|c|c|c|}
\cline { 2 - 3 } \multicolumn{1}{c|}{} & \multicolumn{2}{c|}{ Max Activities per hour } \\
\hline Scenario & $\begin{array}{c}\text { With Camera } \\
\text { Optimization }\end{array}$ & $\begin{array}{c}\text { Without Camera } \\
\text { Optimization }\end{array}$ \\
\hline Scenario 1 & 54 & 45 \\
\hline Scenario 2 & 51 & 42 \\
\hline Scenario 3 & 10 & 9 \\
\hline Scenario 4 & 12 & 11 \\
\hline
\end{tabular}

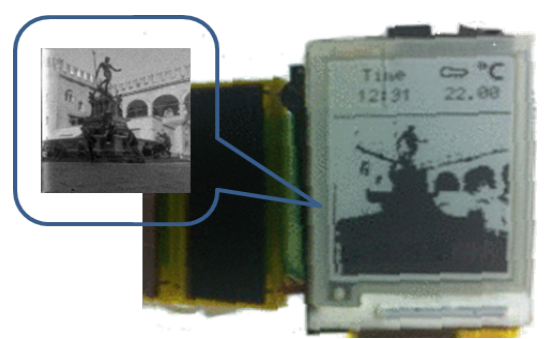

Figure 8: Image captured with camera and visualized on the wearable device.

with and without camera optimization to highlight the importance of the hardware/firmware optimization in terms of energy efficiency. We assume the average value of intake energy is fixed as well as the period of time. In our evaluation, the intake energy $E_{h}$ is the averaged energy accumulated in one hour by the harvester (0.6J). Moreover, we assume the same numbers of acquisition and display, so $\left(A_{e v 1}=A_{e v 2}\right)$, so the consumed energy by the device $E_{\text {consumed }}$ is calculated in one hour. As the intake energy is the same for all the scenarios, bigger is this number of $A_{e v 1}$ and $A_{e v 2}$, better is the energy efficiency of device in the considered scenario. In scenario 1, the image is acquired (with and without optimization) with the camera and the monochrome value is visualized on EPD (Figure 8). In scenario 2, the acquired image is displayed on EPD in 5 grayscale levels. On the other scenarios, 3 and 4, the acquired images are displayed on EPD with 7 and 9 grayscale levels respectively.

Table 3 shows the results for all the eight combinations based on equation 3 and equation 2 with the above mentioned values and assumptions. It is possible to notice that due to the low power design of the wearable device and the combinations of high efficient energy harvesting, our system achieved energy neutrality for all the configurations with a minimal frame rate of 9 acquisitions per hour.

However, Table 3 also shows the benefits of the firmware optimizations. In fact the maximum number of activities per one hour is 54 in scenario 1 , which is 6 times higher than in scenario 3 without firmware optimization. This makes our solutions suitable for a energy-neutral wearable camera which can take almost 1 picture per minute.

\section{CONCLUSIONS}

In this paper we presented an optimized energy neutral wearable camera with a novel electrophoretic display. It is equipped with solar energy harvester, which can significantly extend the lifetime and make it possible to achieve energy neutrality even in challenging indoor scenarios. Power management and optimization strategies on camera and black and white electrophoretic displays are presented to demonstrate the energy consumption reduction of $91.4 \%$ for the camera and $43.7 \%$ for EPD display. We have also enhanced the EPD display with the generation of grayscale images and achieved from 5 to 9 gray levels. Experimental results, based on in-field measurements on power consumption and indoor 
energy harvested, have confirmed the energy neutrality of the device even in indoor scenarios where the device can acquire and display on EPD up to 54 images per hour. This is the first energy neutral smart device with image capturing and displaying capability. The simulation demonstrates the benefits of the aggressive optimizations, that increase the images sampled and displayed from 9 per hour to 54 frames acquired/displayed per hour. With these values the prototype is read to be used as a wearable object to acquire perpetually images.

\section{ACKNOWLEDGMENTS}

This work was supported by the FP7 project PHIDIAS (g.a. 318013) and by Swiss National Science Foundation Projects project Transient Computing (g.a. 200021_157048) and project SCOPES (g.a. IZ74Z0_160481).

\section{REFERENCES}

[1] S. Movassaghi, M. Abolhasan, J. Lipman, D. Smith, and A. Jamalipour, "Wireless body area networks: A survey," Communications Surveys Tutorials, IEEE, vol. 16, no. 3, pp. 1658-1686, Third 2014.

[2] Z. Yanqing, Z. Fan, Y. Shakhsheer, J. Silver, A. Klinefelter, M. Nagaraju, J. Boley, J. Pandey, A. Shrivastava, E. Carlson, A. Wood, B. Calhoun, and B. Otis, "A batteryless $19 \mu \mathrm{w}$ mics/ism-band energy harvesting body sensor node soc for exg applications," Solid-State Circuits, IEEE Journal of, vol. 48, no. 1, pp. 199-213, Jan 2013.

[3] A. Dementyev, J. Gummeson, D. Thrasher, A. Parks, D. Ganesan, J. Smith, and A. Sample, "Wirelessly powered bistable display tags," in Proceedings of the 2013 ACM Int. Joint Conf. on Pervasive and Ubiquitous Computing, ser. UbiComp '13. New York, NY, USA: ACM, 2013, pp. 383-386.

[4] D. Porcarelli, I. Donati, J. Nehani, D. Brunelli, M. Magno, and L. Benini, "Design and implementation of a multi sensors self sustainable wearable device," in Education and Research Conference (EDERC), 2014 6th European Embedded Design in, Sept 2014, pp. 16-20.

[5] A. Mayberry, P. Hu, B. Marlin, C. Salthouse, and D. Ganesan, "ishadow: Design of a wearable, real-time mobile gaze tracker," in Proceedings of the 12th Annual Int. Conf. on Mobile Systems, Applications, and Services, ser. MobiSys '14. New York, NY, USA: ACM, 2014, pp. 82-94.

[6] L. Yoonmyung, B. Suyoung, L. Inhee, K. Yejoong, K. Gyouho, M. Ghaed, P. Pannuto, P. Dutta, D. Sylvester, and D. Blaauw, "A modular $1 \mathrm{~mm}^{3}$ die-stacked sensing platform with low power $\mathrm{i}^{2} \mathrm{c}$ inter-die communication and multi-modal energy harvesting," Solid-State Circuits, IEEE Journal of, vol. 48, no. 1, pp. 229-243, Jan 2013.

[7] J. J. B. Comiskey, J.D. Albert, "Electrophoretic ink: a printable display material," in Digest of Tech. Papers. Soc. Information Display, 1997, pp. 75-76.

[8] Y. Lu and C. Tien, "Principal component analysis of multi-pigment scenario in full-color electrophoretic display," Journal of Display Technology, vol. 9, no. 10, pp. 807-813, 2013.

[9] P. Bai, R. Hayes, M. Jin, L. Shui, Z. Yi, L. Wang, X. Zhang, and G. Zhou, "Review of paper-like display technologies (invited review)," Progress In Electromagnetics Research, vol. 147, pp. 95-116, 2014.

[10] D. Balsamo, A. Weddell, G. Merrett, B. Al-Hashimi, D. Brunelli, and L. Benini, "Hibernus: Sustaining computation during intermittent supply for energy-harvesting systems," Embedded Systems Letters, IEEE, vol. 7, no. 1, pp. 15-18, March 2015.

[11] B. Pires, M. Devyver, A. Tsukada, and T. Kanade, "Unwrapping the eye for visible-spectrum gaze tracking on wearable devices," in Applications of Computer Vision (WACV), 2013 IEEE Workshop on, Jan 2013, pp. 369-376.

[12] E. Campo, D. Hewson, C. Gehin, and N. Noury, "Theme d: Sensors, wearable devices, intelligent networks and smart homecare for health," vol. 34, no. 1, pp. 11 - 13, 2013, digital Technologies for Healthcare.

[13] World Health Organisation, "Health Topics: Disabilities," 2013.

[14] J. Khan, M. R. Yuce, G. Bulger, and B. Harding, "Wireless body area network (wban) design techniques and performance evaluation," Journal of medical systems, vol. 36, no. 3, pp. 1441-1457, 2012.

[15] K. Baskaran, "A survey on futuristic health care system: Wbans," Procedia Engineering, vol. 30, pp. 889-896, 2012.

[16] C. Perera, A. Zaslavsky, P. Christen, and D. Georgakopoulos, "Context aware computing for the internet of things: A survey," Communications Surveys Tutorials, IEEE, vol. 16, no. 1, pp. 414-454, First 2014.

[17] C. Pang, C. Lee, and K. Suh, "Recent advances in flexible sensors for wearable and implantable devices," Journal of Applied Polymer Science, vol. 130, no. 3, pp. 1429-1441, 2013.

[18] M. Gorlatova, J. Sarik, G. Grebla, M. Cong, I. Kymissis, and G. Zussman, "Movers and shakers: Kinetic energy harvesting for the internet of things," in The 2014 ACM Int. Conf. on Measurement and Modeling of Computer Systems, ser. SIGMETRICS '14. New York, NY, USA: ACM, 2014, pp. 407-419.

[19] P. Castillejo, J. Martínez, L. López, and G. Rubio, "An internet of things approach for managing smart services provided by wearable devices," International Journal of Distributed Sensor Networks, vol. 2013, 2013.

[20] M. Magno, D. Porcarelli, D. Brunelli, and L. Benini, "Infinitime: A multi-sensor energy neutral wearable bracelet," in Green Computing Conference (IGCC), 2014 International. IEEE, 2014, pp. 1-8.

[21] S. Inoue, H. Kawai, S. Kanbe, T. Saeki, and T. Shimoda, "High-resolution microencapsulated electrophoretic display (epd) driven by poly-si tfts with four-level grayscale," Electron Devices, IEEE Transactions on, vol. 49, no. 9, pp. 1532-1539, Sep 2002.

[22] Pervasive Displays Inc, "1.44" TFT EPD Panel (EK014BS011)," 2014.

[23] P. Bai, Z. Yi, and G. Zhou, "An improved driving scheme in an electrophoretic display," International Journal of Engineering and Technology, vol. 3, no. 4, pp. 436-441, 2013.

[24] H. Yee, "Perceptual metric for production testing," Journal of Graphics Tools, vol. 9, no. 4, pp. 33-40, 2004.

[25] M. Baker, A. Shrivastava, and K. Chatha, "Smart driver for power reduction in next generation bistable electrophoretic display technology," in CODES+ISSS, 2007 5th IEEE/ACM/IFIP International Conference on, Sept 2007, pp. 197-202. 